\title{
Complementary and Alternative Medicine use in patients with head and neck cancers in Europe
}

\begin{abstract}
Alexander Molassiotis ${ }^{1}$, Gulten Ozden², Nurgun Platin ${ }^{3}$, Julia A. Scott ${ }^{4}$, Dorit Pud $^{5}$, Paz Fernandez-Ortega ${ }^{6}$, Ljiljana Milovics ${ }^{7}$, Vassiliki Panteli ${ }^{8}$, Gudbjorg Gudmundsdottir9, Maria Browal ${ }^{10}$, Elin Madsen ${ }^{11}$, Miriam Magri ${ }^{12}$, Anita Margulies $^{13}$, Sarka Selvekerova ${ }^{14}$, Nora Kearney ${ }^{4}$, Elisabeth Patiraki ${ }^{8}$.
\end{abstract}

${ }^{1}$ School of Nursing, University of Manchester, UK

${ }^{2}$ Gazi University Hospital, Ankara, Turkey

${ }^{3}$ School of Health Sciences, Koc University, Istanbul, Turkey

${ }^{4}$ Department of Nursing and Midwifery, University of Stirling, Stirling, Scotland

${ }^{5}$ Faculty of Social Welfare and Health Studies, University of Haifa, Haifa, Israel.

${ }^{6}$ Institut Català Oncologia ICO, Barcelona, Spain

${ }^{7}$ Department of Education, Institute for Oncology and Radiology, Belgrade, Serbia and Montenegro

${ }^{8}$ Greek Oncology Nursing Society, Athens, Greece

${ }^{9}$ Department of Oncology, Lanspitali, Reykjavik, Iceland

${ }^{10}$ Sahlgrenska University Hospital, Gothenburg, Sweden

${ }^{11}$ Oncology Department, Aarhus University Hospital, Aarhus, Denmark

${ }^{12}$ Università degli Studi di Milano- Istituto Nazionale Tumori, Milan, Italy

${ }^{13}$ Zurich University Hospital University Hospital, Poliklinik Onkologie, Zurich, Switzerland

${ }^{14}$ Masaryk Memorial Cancer Institute, Brno, Czech Republic

Address for correspondence:

Alexander Molassiotis, RN, PhD

Reader in Cancer \& Supportive Care 
University of Manchester

School of Nursing

Coupland III

Coupland Street

MANCHESTER M13 9PL

email: alex.molassiotis@man.ac.uk

tel: +44 (0)161-2755337

fax: +44 (0)161-2757566 


\begin{abstract}
AIM: The aim of the present study was to examine the patterns of complementary and alternative medicine (CAM) use in a sample of head and neck cancer patients, forming part of a larger study. METHODS: A crosssectional survey design was used collecting data through a descriptive 27-item questionnaire in 12 countries in Europe. RESULTS: The participants were 75 patients with head and neck cancers. The prevalence rate of CAM use was $22.7 \%$. The most common therapies used were herbal medicine (47\%), medicinal teas (23.5\%), use of vitamins/minerals (11.8\%) and visualisation $(11.8 \%)$. Use of CAM dramatically increased after the diagnosis with cancer (ie. 8-fold increase in the use of herbs). A profile of CAM user was not evident in this sample. Patients used CAM for a variety of reasons together, with counteracting the ill effects from cancer and its treatment being the most common one. Information about CAM was obtained mostly from friends and family. CONCLUSIONS: As one in five head and neck cancer patients use CAM it is important that clinicians explore practices with their patients, improve communication about CAM with them and assist those who want to use CAM in using appropriate and safe therapies.
\end{abstract}

Key words: complementary medicine, alternative medicine, complementary therapies, head and neck cancer. 


\section{Complementary and Alternative Medicine use in patients with head and neck cancers in Europe}

\section{Introduction}

It is evident from the literature that a significant number of cancer patients turn to complementary and alternative medicine (CAM) as adjunct therapies to their cancer treatment $[1,2,3]$. Prevalence of use is as high as $80 \%$ in some studies [4] although a systematic review of 26 surveys in 13 countries suggests that at least one-third of cancer patients (31.4\%) are using some form of CAM after the diagnosis of cancer [5]. In a UK-based survey, lifetime use of CAM was reported to be $46.6 \%$, whereas CAM use in the past 12 months was lower at $28.3 \%$ [6]. The literature also suggests that female gender, higher education level and younger age are commonly predicting CAM use $[4,7,8]$. However, most work derives from American samples of breast cancer patients $[9,10,11]$ and studies assessing CAM use in breast cancer patients may not be representative of CAM use in other cancer diagnostic groups.

No studies were found in the literature assessing CAM use specifically in head and neck cancer patients. However, one such study used a mixed sample of cancer patients $(n=453)$ in which 52 patients with thoracic/head and neck cancer were also included [7]. In the latter study, $68.7 \%$ of patients had used at least one CAM modality, a number that increased to $83.3 \%$ if psychotherapy and spiritual practices were included. Spiritual practices, vitamins/herbs, movement/ physical therapies and mind/body therapies were the most common CAM modalities used. Younger patients were 2.1 times more likely to use CAM than older ones and female patients were 1.8 times more likely to use CAM than male patients. Feeling more hopeful was the most common reason cited $(73 \%)$ for using CAM [7].

The aim of the present study was to assess the patterns of CAM use in a sample of head and neck cancer patients in Europe. The present report forms a 
subgroup analysis of head and neck cancer patients drawn from a larger $(n=$ 956) European survey of CAM use in cancer patients [12].

\section{Methods}

The present study was a descriptive cross sectional survey carried out in 14 countries in Europe with 12 countries contributing data from head and neck cancer patients. A member of the Oncology Nursing Society in each country facilitated the study locally. The 12 participating countries included Turkey, Greece, the United Kingdom, Israel, Spain, Serbia, Iceland, Sweden, Denmark, Italy, Switzerland and the Czech Republic. The study was approved by the Research and Ethics Committee of all participating hospitals. Data was collected from all cancer patients attending an outpatient clinic on randomly selected days.

A descriptive questionnaire was used to collect the data based on the one developed by Swisher et al [13], modified for the needs of the present study. It consisted of 27 items. The questionnaire was translated in to all the languages of the participating countries before use and was standardised. Its first section enquired about sociodemographic characteristics and medical data. Following that, patients were asked whether they used CAM before or after the diagnosis of cancer. If they responded negatively, they were asked to select from a list the main reason for that. If they responded positively, a number of questions followed enquiring about the type(s) of CAM used, the reasons for using CAM and benefits experienced, expenditure on CAM and sources of information about CAM. Furthermore, a 7-point scale was used to assess satisfaction with the CAM used and another 7-point scale to assess perceived effectiveness of the CAM used, with higher scores representing more satisfaction and higher level of perceived effectiveness respectively. A detailed description of the methods is presented elsewhere [12].

Data was coded and analysed using the Statistical Package for the Social Sciences (SPSS). Descriptive statistics were used with all variables to 
summarise the data. Non parametric tests were used to assess any differences between sociodemographic or clinical data and use of CAM. Correlation coefficients were also calculated.

\section{Results}

Each country contributed in relation to head \& neck cancer patients with $1.3 \%$ to $30.7 \%$ of the overall data. However, four countries contributed with $69.4 \%$ of the data, including Turkey (30.7\%), the United Kingdom (16\%), Israel (12\%) and Spain $(10.7 \%)$.

The sample consisted of 75 patients with head and neck cancers. There were 46 male patients (61.3\%) and 29 (38.7\%) female patients. Most were of low educational level (ie. only primary school $=43.2 \%$ ) and manual workers $(31.5 \%)$. Income in $52.8 \%$ of the sample was less than $€ 10,000$ annually. Most were married $(66.7 \%)$ and all but $2(2.6 \%)$ were white Europeans/Caucasians (Table 1). At the time the study took place, $70.7 \%$ were receiving (conventional) treatment for their cancer, with chemotherapy accounting for $66 \%$ of the treatments received.

\section{INSERT TABLE 1 HERE}

Seventeen patients $(22.7 \%)$ reported use of CAM before or after the diagnosis of cancer. Nine therapies were used before the diagnosis of cancer, 12 therapies were used since the diagnosis of cancer ( 8 of them were new therapies never used before the cancer diagnosis) and 10 therapies were currently used. The most common CAM therapies used since the diagnosis of cancer were herbal medicines $(47.1 \%)$, medicinal teas $(23.5 \%)$, vitamins/minerals $(11.8 \%)$ and visualisation (11.8\%) (Table 2). Use of herbal remedies showed a dramatic increase after the diagnosis of cancer by almost 8 times, whereas use of medicinal teas increased four-fold. Patients spent an average of $€ 260.57$ per month $(S D=345.9)$, with a great variation from patient to patient varying from 
$€ 14$ to $€ 987$ per month. Patients were relatively satisfied with the use of CAM ( mean $=5.06, S D=2$ ) although they felt that CAM was moderately effective (mean $=4.40, S D=1.68)$. There were no statistical differences between any of the sociodemographic characteristics of the sample and use of CAM. However previous treatment for cancer was a predictor of CAM use $(p=0.016)$

\section{INSERT TABLE 2 HERE}

Users of CAM tended to be more satisfied with CAM use if they also realised improvements in their physical well being $\left(r_{s}=0.55, p<0.05\right)$ or emotional well being and hope/optimism $\left(r_{s}=0.56, p<0.005\right)$. Higher satisfaction was generally associated with higher perception of CAM effectiveness $\left(r_{s}=0.86, p<0.001\right)$.

Participants used CAM for more than one reason at the same time, mostly to counteract ill effects from cancer or its treatment (41.2\%), to do everything possible to fight the cancer (35.3\%), because it may help (35.3\%) or to improve their emotional well being, hope and optimism (35.3\%). Almost a quarter of the patients $(23.5 \%)$ found no benefit from using CAM, especially in relation to counteracting ill effects from the cancer or its treatment $(p<0.05)$, although they realised an overall improvement in their physical well being $(p<0.05)$ (Table 3$)$. Two patients (11.8\%) reported side effects from using CAM, and both were using stinging nettle teas as a herbal remedy.

Friends were the most common source of information about CAM (47.1\%), followed by family (29.4\%), the media (newspapers, books, tv, radio) in $23.5 \%$ or CAM practitioners $(23.5 \%)$. Other less frequently reported sources of information included physicians $(11.8 \%)$, other patients $(5.8 \%)$ or the internet $(5.8 \%)$. None reported the nurse as a source of CAM information.

Those participants who reported no use of CAM were asked to select the main reason for that from a list of choices. The majority (48.9\%) were satisfied with 
the (conventional) treatment they received/were receiving. One-third of patients $(36.2 \%)$ had never thought of CAM and another $4.3 \%$ that they did not use CAM because of the lack of information about CAM effectiveness. Two patients $(4.3 \%)$ were discouraged by family to use CAM, one $(2.1 \%)$ could not afford to pay for CAM and one patient was interested in CAM but had not decided to do so as yet.

\section{Discussion}

The present study is the first report of CAM use specifically in relation to head and neck cancer. This group of patients is a particularly "difficult" group to manage clinically, partly because of the poor prognosis of the disease and its sociodemographic characteristics (i.e. low education level). Hence, information about CAM use in this group of patients may shed light in other practices used outside the conventional health care setting by the patients. The data suggests that only $22.7 \%$ of the patients used CAM, a prevalence rate well below the mean rate of the larger European study of $36.4 \%$ [12] and the international literature $[1,7,14,15]$.

The range of CAM therapies used was also limited and only few of the CAM therapies tried before the cancer diagnosis were used after the diagnosis with cancer, most being new therapies. By far the most commonly used CAM therapy was herbal medicine, increasing eight-fold from before the cancer diagnosis. The most common herbs used was nettle teas or mixtures using dry nettle or nettle seeds, although a large number of herbs used were not identified (many patients did not know the names of the herbs used). Other biological-based therapies were also common. As head and neck cancers have a poor prognosis and they are facing many debilitating symptoms in everyday life, it would be interesting to explore in future studies whether such biologically-based methods have any effects in symptom management but also in improving quality of life. Reports in the literature suggest that cancer patients who use CAM demonstrate significant improvements in their psychosocial status compared to non-users 
[16]. CAM may have a significant role to play, especially in supportive and palliative care.

There was no report of use of any manipulative therapies (i.e. osteopathy or chiropractic). Only a small percentage of spiritual or relaxation therapies (with the exception of visualisation) were reported, which are some of the most commonly used therapies as reported in the literature $[7,15,17]$. The average of 1.3 CAM therapies used per person is also considerably lower from the 4.8 therapies reported in the study by Richardson et al [7], suggesting a less active coping style or a more passive and pessimistic attitude.

Although the literature suggests that the typical profile of the CAM user is that of a young, female and better educated person $[7,18,19]$, this was not shown in the present study. As much of the international literature has been conducted with breast cancer patients, a typical user profile may not be the case in other cancer diagnostic groups, especially those with poor prognosis. Furthermore, the patients in the current study spent a large amount of money for CAM therapies on remedies each month which was an average of $€ 260$, significantly more than the average spending of $\$ 68$ annually reported by Patterson et al [8]. It would be interesting in future studies to explore out-of-pocket expenses in cancer patients with good and poor prognosis, as the latter group may be spending more money in order to increase their chances of longer survival.

A variety of reasons for using CAM was reported, with counteracting side effects being the most common. A significantly lower number of patients reported benefits in relation to counteracting side effects or directly fighting the disease compared to the initial reason for CAM use, supporting past results [13]. However, significantly more benefits compared to the initial reason for using CAM were reported in relation to improvements in physical well being. Nevertheless, almost a quarter of patients reported no benefits from the CAM therapies used and a considerable number of side effects $(11.8 \%)$ were reported. 
This suggests that head and neck cancer patients need education, advise and guidance about which therapies may potentially be useful for them. Such discussions have also the potential to improve overall communication with the patients. Also, cancer patients may be motivated to use CAM in order to increase hope and optimism to cope with a devastating illness and this may be the key motivator for patients. Ritvo et al [20] explored this assertion using the Risk Adaptation Model to explain the motivation to use CAM and also describe the cognitive process for cancer patients deciding on the use of CAM.

Friends, family and the media were the main sources of information in this group of patients, confirming earlier reports [21]. This is informal and uncontrolled information, often not based on evidence but rather personal testimonials. This further reiterates the need for more credible and appropriate information to be available to patients, and health professionals can play a major role in this area. As some clinicians may find it challenging to talk to patients about CAM due to their own limited knowledge on the topic, the step-by-step strategy for responding to patients questions suggested by Eisenberg [22] may be a useful aid.

A limitation of the study is the relatively small sample size used. Also, small numbers of patients participating from a number of countries may have skewed some results, even though the larger European study [12] did not show many major differences in the CAM use across countries. Future research should explore how head and neck cancer patients come to select a given CAM therapy, for what specific purpose and how this helps them (if it does) to better cope with their illness and often aggressive surgery. 


\section{References}

1. DiGianni LM, Garber JE, Winer EP. Complementary and alternative medicine use among women with breast cancer. J Clin Oncol 2002, 20(18s): 34s-38s.

2. Sparber A. Wootton JC. Surveys of complementary and alternative medicine: Part II. Use of alternative and complementary cancer therapies. J Altern Compl Med 2001, 7:281-287.

3. Jacobson JS, Verret WJ. Complementary and alternative therapy for breast cancer. The evidence so far. Cancer Pract 2001, 9: 307-310.

4. Bernstein BJ, Grasso T. prevalence of complementary and alternative medicine use in cancer patients. Oncol (Hunt) 2001, 15: 1267-1272.

5. Ernst E. Cassileth BR. The prevalence of complementary/alternative medicine in cancer: a systematic review. Cancer 1998, 83:777-782.

6. Thomas KJ, Nicholl JP, Coleman P. Use and expenditure on complementary medicine in England. A population based survey. Compl Ther Med 2001, 9: 211.

7. Richardson MA, Sanders T, Palmer JL, Greisinger A, Singletary SE. Complementary/alternative medicine use in a comprehensive cancer center and the implications for oncology. J Clin Oncol 2000, 18: 2505-2514.

8. Patterson RE, Neuhouser ML, Hedderson MM, Schwartz SM, Standish LJ, Bowen DJ, Marshall LM. Types of alternative medicine used by patients with breast, colon, or prostate cancer: predictors, motives, and costs. J Altern Compl Med2002, 8: 477-485.

9. Burstein HJ, Gelber S, Guadagnoli E, Weeks JC. Use of alternative medicine by women with early-stage breast cancer. New Engl J Med 1999, 340: 17331739.

10. Lee MM, Lin SS, Wrensch MR, Adler SR, Eisenberg D. Alternative therapies used by women with breast cancer in four ethnic populations. J Natl Cancer Inst 2000, 92: 42-47.

11. VandeCreek L, Rogers E, Lester J. Use of alternative therapies among breast cancer outpatients compared with the general population. Altern Ther Health Med 1998, 5: 71-76. 
12. Molassiotis A, Fernadez-Ortega P, Pud D, et al. Use of complementary and alternative medicine in cancer patients: a European survey. Eur J Cancer (submitted for publication).

13. Swisher EM, Cohn DE, Goff BA, Parham J, Herzog TJ, Rader JS, Mutch DG. Use of complementary and alternative medicine among women with gynaecologic cancers. Gyn Oncol 2002, 84: 363-367.

14. Paltiel O, Avitzour M, Peretz T, Cherny N, Kaduri L, Pfeffer RM, Wagner N, Soskolne V. Determinants of the use of complementary therapies by patients with cancer. J Clin Oncol 2001, 19: 2439-2448.

15. Morris KT, Johnson N, Homer L, Walts D. A comparison of complementary therapy use between breast cancer patients and patients with other primary tumor sites. Am J Surg 2000, 179: 407-411.

16. Gilbar O, Iron G, Goren A. Adjustment to illness of cancer patients treated by complementary therapy along with conventional therapy. Patient Educ Couns 2001, 44: 243-249.

17. Rees RW, Feigel I, Vickers A, Zollman C, McGurk R, Smith C. Prevalence of complementary therapy use by women with breast cancer: a population based survey. Eur J Cancer 2000, 36: 1359-1364.

18. Shumay DM, Maskarinec G, Gotay CC, Heiby EM, Kakai H. Determinants of the degree of complementary and alternative medicine use among patients with cancer. J Altern Compl Med 2002, 8: 661-671.

19. Gray RE, Fitch M, Goel V, Franssen E, Labrecque M. Utilization of complementary/alternative services by women with breast cancer. J Health Soc Policy 2003, 16: 75-84.

20. Ritvo P, Irvine J, Katz J, Matthew A, Sacamano MA, Shaw BF. The patient's motivation in seeking complementary therapies. Patient Educ Couns 1999, 38: 161-165.

21. Shen J, Andersen R, Albert PS, Wenger N, Glaspy J, Cole M, Shekelle P. Use of complementary/alternative therapies by women with advanced-stage breast cancer. BMC Compl Altern Med 2002, 2:8. 
22. Eisenberg D. Advising patients who seek alternative medical therapies. Ann Intern Med 1997, 127: 61-69. 
Table 1. Sociodemographic data of the sample

\begin{tabular}{|l|l|l|}
\hline & & $\mathbf{N}^{*}(\%)$ \\
\hline Gender & Male & $46(61.3)$ \\
& Female & $29(38.7)$ \\
\hline Marital Status & Single & $14(18.7)$ \\
& Married & $50(66.7)$ \\
& Divorced/separated & $8(10.6)$ \\
& Widowed & $3(4)$ \\
\hline Educational level & Primary school & $32(43.2)$ \\
& Secondary (high) school & $28(37.9)$ \\
& College/University degree & $11(14.9)$ \\
& Postgraduate education & $3(4)$ \\
\hline Occupation & Retired & $20(27.4)$ \\
& Education/business & $9(12.3)$ \\
& Housewives & $14(19.2)$ \\
& Manual work & $23(31.5)$ \\
& Clerical staff & $1(1.4)$ \\
& Unemployed & $6(8.2)$ \\
\hline Annual Income (in $€)$ & $<10,000$ & $28(52.8)$ \\
& $10,001-20,000$ & $14(26.4)$ \\
& $20,001-30,000$ & $5(9.4)$ \\
& $30,001-40,000$ & $3(5.7)$ \\
& $>40,000$ & $3.7)$ \\
\hline
\end{tabular}

* Numbers do not add up all to total sample size, as there was some missing data. 
Table 2. Types and frequency of complementary and alternative medicine used by head and neck cancer patients [N(\%)]

\begin{tabular}{|c|c|c|c|c|c|c|}
\hline \multirow{2}{*}{ Type } & \multirow{2}{*}{\multicolumn{2}{|c|}{ Before Cancer }} & \multirow{2}{*}{\multicolumn{2}{|c|}{$\begin{array}{l}\text { Since diagnosis } \\
\underline{\text { With Cancer }}\end{array}$}} & \multirow{2}{*}{\multicolumn{2}{|c|}{ Currently }} \\
\hline & & & & & & \\
\hline Vitamins/minerals & 1 & (5.9) & 2 & $(11.8)$ & 3 & $(17.6)$ \\
\hline Homeopathy & 1 & (5.9) & 1 & (5.9) & & \\
\hline Herbalmedicine & 1 & $(5.9)$ & 8 & $(47.1)$ & 7 & $(41.2)$ \\
\hline Medicinal teas & 1 & $(5.9)$ & 4 & $(23.5)$ & 4 & $(23.5)$ \\
\hline Acupuncture & 1 & $(5.9)$ & & & & \\
\hline Reflexology & 1 & $(5.9)$ & & & 2 & $(11.8)$ \\
\hline Oxygen therapy & 1 & $(5.9)$ & & & & \\
\hline Yoga & 1 & $(5.9)$ & & & & \\
\hline Shiatsu & 1 & $(5.9)$ & & & 1 & (5.9) \\
\hline Spiritual therapies & & & 1 & $(5.9)$ & 1 & $(5.9)$ \\
\hline Aromatherapy & & & 1 & $(5.9)$ & 1 & (5.9) \\
\hline Juicing & & & 1 & $(5.9)$ & 1 & (5.9) \\
\hline Electromagnetic therapy & & & 1 & (5.9) & 1 & $(5.9)$ \\
\hline Massage & & & 1 & (5.9) & & \\
\hline Visualisation & & & 2 & $(11.8)$ & & \\
\hline Hypnotherapy & & & 1 & $(5.9)$ & & \\
\hline Support Groups & & & 1 & $(5.9)$ & 1 & (5.9) \\
\hline
\end{tabular}


Table 3. Reasons for using complementary and alternative therapies and perceived benefits $[\mathrm{N}(\%)]$

Reasons for Use

To directly fight the disease

To increase body's ability to fight the cancer

To improve physical well-being

To improve emotional well-being,

hope and optimism

To counteract ill effects

"Help, no hurt"

To do everything to fight the disease

Requested by the physician

${ }^{*} \mathrm{P}<0.05$
Benefits experienced

$2(11.8)^{*}$

$5 \quad(29.4)$

$9 \quad(52.9)^{*}$

6

$6 \quad(35.3)$

4

$(23.5)^{*}$ 